\title{
ASCO 2019: New Results in Breast Cancer
}

\author{
Chair: Oleg Gluza \\ Participants: Andreas Hartkopf ${ }^{\mathrm{b}}$ Sherko Kümmelc Frederik Marméd $^{\mathrm{c}}$ \\ a Johanniter GmbH - Evangelisches Krankenhaus Bethesda Mönchengladbach, West German Study Group,

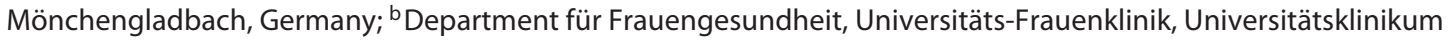 \\ Tübingen, Tübingen, Germany; ${ }^{c}$ Klinik für Senologie / Interdisziplinäres Brustkrebszentrum, KEM | Ev. Kliniken \\ Essen-Mitte, Ev. Huyssens-Stiftung Essen-Huttrop, Essen, Germany; ${ }^{\mathrm{d}}$ Frauenklinik, Universitätsklinikum Mannheim, \\ Medizinische Fakultät Mannheim der Universität Heidelberg, Mannheim, Germany
}

\section{Question 1: What Is the Most Promising Treatment De-Escalation Tool in the Early HER2-Positive Breast Cancer?}

Hartkopf: Neoadjuvant chemotherapy enables "in situ" prediction of treatment efficacy, and patients who achieve a pathological complete response (pCR) have a decreased risk for distant relapse and death from breast cancer. Although biomarkers (e.g., gene signatures, HER2 expression, immune markers) can also help predict treatment efficacy and may therefore help de-escalate treatment, tumor heterogeneity challenges their use. Hence, in my opinion, response to neoadjuvant treatment is the most promising marker to tailor therapy with a de-escalation of treatment in patients who do achieve a pCR and further characterization of residual tumor (i.e., therapy-resistant tumor clones) to specifically tailor therapy in patients who do not.

Kümmel: The most promising de-escalation tool after the data by Sara Tolaney with paclitaxel mono over 12 weeks and trastuzumab is T-DM1 as an antibody-drug conjugate (ADC) targeted the Her2/neu pathway. Thirty to fifty percent of the patients have a $\mathrm{pCR}$, and as we know now from the KRISTINE trial, this pCR is a surrogate for overall survival comparable to a standard chemotherapy with dual blockage (trastuzumab + pertuzumab). With this data, a minimum of every 3 rd patient has no hair loss; e.g., can go to work during chemotherapy etc. and possibly need no further chemotherapy after 4-6 cycles of T-DM1. Open questions would be the best therapy after $\mathrm{pCR}$ and nonpCR in this fashion. These could be a backbone of new trials with ADCs focusing on Her2 overexpression. Further- more, these data are supported by the ADAPT triple-positive trial. Interesting could be the translational research program from these trials, if it is possible according to tumor-infiltrating lymphocytes, Her2/neu-enriched status, and different immune markers or gene signatures to select patients with a high chance of pCR. Another important point in the biomarker discussion would be the analysis of Her2/neu heterogeneity in the tumor and its impact on static or dynamic markers (after 2 cycles of therapy).

Marmé: In principal, there are two major options for treatment de-escalation, which avoid "free" chemotherapy: dual HER2 blockade with trastuzumab/pertuzumab or the ADC T-DM-1. The best way to implement these strategies is the neoadjuvant setting as this allows for a backup strategy for patients not achieving a pCR; e.g., standard chemotherapy plus HER2-targeted therapy. Both approaches have been investigated in neoadjuvant trials but have not been compared directly. Important in the development of such strategies is to investigate predictive factors (e.g., HR status for trastuzumab/pertuzumab, HER2 tumor heterogeneity for T-DM1) as well as early response markers (Ki-67 dynamics, PET-CT etc.).

\section{Question 2: What Do You Think about Neoadjuvant/ Adjuvant T-DM1 Data?}

Hartkopf: The KATHERINE trial is the first randomized trial that demonstrates a clear benefit of using an antibody-drug conjugate (T-DM1) in HER2-positive patients who do not achieve a $\mathrm{pCR}$ during neoadjuvant

\section{KARGER}

(C) 2019 S. Karger AG, Basel 
treatment. Maybe even more important is the fact that it is a proof a concept for tailoring treatment using response to neoadjuvant treatment. The KATHERINE trial is, however, an "escalation trial." The next step would be to de-escalate therapy in patients who do achieve a pCR.

Kümmel: Data from the KATHERINE study switching to T-DM1 are practice changing for patients who did not have pCR after chemotherapy and antibody therapy in the Her2/neu-positive situation.

Especially in view of the data of the ADPAT triplepositive and KRISTINE studies, a de-escalation with T-DM1 in the neoadjuvant situation would be conceivable and certainly a possible concept with upcoming ADCs that are directed to the Her2/neu pathway.

Marmé: Briefly, T-DM1 as post-neoadjuvant therapy in non-pCR patients with HER2-positive disease is a clear standard. The neoadjuvant T-DM1 data presented at the ASCO appears highly interesting, even if the pCR rates and event-free survival are inferior to the standard arm in KRISTINE. Importantly, patients achieving a pCR on T-DM1 have exactly the same excellent prognosis as those treated with standard chemotherapy plus trastuzumab and pertuzumab. The pCR rate on T-DM1 was $44 \%$, which suggests that nearly half the patients could benefit from this strategy avoiding standard chemotherapy. HER2 intratumor heterogeneity seems to be a very promising biomarker to identify patients who are not candidates for this approach and might help select patients.

\section{Question 3: What Do You Recommend Doing (Use or Not to Use Genomic Markers) in Clinical High-Risk HR+/HER2- Node-Negative Premenopausal Disease regrading Treatment Escalation (Chemotherapy and/or Extended Endocrine Therapy)?}

Hartkopf: At this year's ASCO annual meeting, a prespecified analysis of the TAILORx trial was presented to evaluate whether clinical risk provides additional prognostic information in patients with primary HR+/HER2breast cancer. The authors found that clinical risk can help guide chemotherapy recommendation in women $<50$ years old and a recurrence score (RS) of 16-25. Additionally, data from the PlanB trial showed that clinical high-risk patients with a low RS $(<11)$ have an excellent prognosis even without chemotherapy. Conversely, an important question that cannot be answered by the TAILORx study is whether clinical low-risk patients with a high-risk RS do benefit from chemotherapy. Hence, I would use genomic markers but always integrate clinical risk to discuss rather than recommend different treatment options on an individual basis.

New Results in Breast Cancer from ASCO
Kümmel: This is one of the most challenging tasks after presenting the TAILORx study data in relation to a classical clinical-risk profile. I would also advocate additional molecular genetic analysis in premenopausal patients; e.g., to identify the group with a RS $<16$ that does not benefit from chemotherapy. Additional chemotherapy has shown an advantage in the molecular genetic intermediate group with a high-risk clinical profile. It should be noted, however, that the effect of chemotherapy may be due to a therapy-induced amenorrhea, which may explain the data, especially in the premenopausal situation. In summary, with the clinical and molecular genetic results, we have a lot of knowledge to be able to advise patients even better and safer in the future, and I would support the deliberate use of molecular genetic testing. These data are in line with the previously known data from the PlanB and ADAPT studies, which also included intermediate-risk lymph-node-positive patients.

Marmé: In node-negative HR+/HER2- early breast cancer, I strongly recommend the use of prognostic multigene signatures in cases in which a reliable assessment of risk of recurrence is impossible based on standard pathologic factors (for HR+/HER2-: mainly grading and Ki-67). In node-negative cases, risk is mainly defined by tumor biology; i.e., by a higher proliferation, grade, or a high-risk gene signature. For such high-risk cases, my first choice for escalation would be the addition of adjuvant chemotherapy. If still or again premenopausal after chemotherapy, ovarian function suppression will play a role. With respect to extended endocrine therapy, the EBCTCG meta-analysis can guide individual decision-making, with little benefit from extended endocrine therapy in node-negative patients having the least benefit, especially if an aromatase inhibitor is given within the first 5 years. For those who remain premenopausal during the 5 years of endocrine therapy, the matter is more complicated and is even more so if $\mathrm{GnRH}$ analogues are used. There is still a lack of data, and this needs to be discussed at an individual basis with the patient, taking into account tolerability and the perceived remaining risk. My definition of clinical risk is based on relevant nodal involvement, high proliferation, and grade.

\section{Question 4: What is Your Definition of Clinical High Risk?}

Hartkopf: The strongest prognostic factor is lymph node involvement. As node-positive patients were not part of the TAILORx study, data from the RxPONDER trial are eagerly awaited to define optimal RS cutoff values in this population. 
Kümmel: Clinical high risk (HR+) regarding an escalated chemoendocrine therapy is an endocrine-insensitive tumor according to the POETIC or ADAPT data - no sufficient Ki-67 decrease in a 2-to-3-week endocrine window phase - and a genomic high-risk score of the tumor additionally to the classical clinical high-risk factors; e.g., lymph node and the amount of expression of the estrogen and progesterone receptor. This would be the patients who may benefit from an extended CDK 4/6 therapy together with endocrine therapy after chemotherapy. These should only be recommended in clinical trials; e.g., the NataLEE trial that starts next month in Germany.

\section{Question 5: What Is the Most Promising Treatment in Metastatic HER2 Disease after Failure of Standard Taxane + Pertuzumab + Trastuzumab and T-DM1?}

Hartkopf: Even though data was presented at this year's ASCO annual meeting showing that neratinib + capecitabine seems more effective than lapatinib + capecitabine, the increased toxicity (diarrhea) in a noncurative setting is challenging. Other HER2-directed antibodies or their combination might slightly enhance therapy efficacy; however, in my opinion, different strategies than targeting the HER2 receptor are needed after failure of trastuzumab, pertuzumab, and T-DM1. Even though there is currently no data showing that immunotherapy is effective in this population, it is a promising option. We must, however, learn which patients are likely to benefit from immunotherapy and how to enhance anti-tumor immune response in patients who do not.

Kümmel: In the advanced therapy line for metastatic breast cancer, the positive data on neratinib in combination with capecitabine are of particular interest at this year's ASCO annual meeting and are a possible therapeutic alternative, whereby the control of the side effects will move into the focus during therapy planning. The previous data on immuno-oncological therapeutic approaches were not as convincing as was hoped, and we must wait for further study results. Additional therapy options will be tested in the Destiny program with the ADC trastuzumab deruxtecan (DS8201).

Marmé: At this moment, the most promising agents in HER2+ metastatic breast cancer after trastuzumab/pertuzumab and T-DM1 to me are second-generation ADCs such as trastuzumab deruxtecan that demonstrate very promising responses in patients resistant to T-DM1. The new data from ASCO are interesting. However, the clinical benefit from margetuximab in the post-trastuzumab/ pertuzumab and post-T-DM1 setting was modest, and the substitution of neratinib for lapatinib in combination with capecitabine was problematic in terms of diarrhea. Several other agents (ADCs and tyrosine kinase inhibitors) are currently in clinical development.

\section{Participants}

Prof. Dr. med. Andreas Hartkopf Department für Frauengesundheit Universitäts-Frauenklinik, Universitätsklinikum Tübingen Calwerstrasse 7, 72076 Tübingen, Germany andreas.hartkopf@uni-tuebingen.de

Prof. Dr. med. Sherko Kümmel Klinik für Senologie / Interdisziplinäres Brustkrebszentrum KEM | Ev. Kliniken Essen-Mitte

Ev. Huyssens-Stiftung Essen-Huttrop

Henricistrasse 92, 45136 Essen, Germany

S.Kuemmel@kem-med.com

Prof. Dr. med. Frederik Marmé

Frauenklinik, Universitätsklinikum Mannheim

Medizinische Fakultät Mannheim der Universität Heidelberg

Theodor-Kutzer-Ufer 1-3, 68167 Mannheim, Germany

Frederik.Marme@umm.de 